\title{
Resonant two-wave mixing in photorefractive materials with the aid of dc and ac fields
}

\author{
V. A. Kalinin \\ School of Engineering, Oxford Brookes University, Gipsy Lane Campus, Headington, Oxford OX3 OBP, UK
}

K. Shcherbin

Institute of Physics, National Academy of Sciences, 46 Science Avenue, 252650 Kiev-22, Ukraine

L. Solymar and J. Takacs

Department of Engineering Science, University of Oxford, Parks Road, Oxford OX1 3PJ, UK

D. J. Webb

Physics Laboratory, University of Kent at Canterbury, Kent CT2 7NR, UK

Received June 19, 1997

\begin{abstract}
A recently predicted resonant effect for the enhancement of two-wave mixing in photorefractive materials is investigated. The resonance occurs when the frequency of the applied ac field agrees with the eigenfrequency of the excited space-charge wave. Experimentally a clear resonance is found, as predicted by the theory, for high dc electric fields, but the resonance is smeared out for lower fields. A modified theory, taking into account the second temporal harmonic of the space-charge wave, shows good agreement with the experimental results. (C) 1997 Optical Society of America
\end{abstract}

It was shown by Huignard and Marrakchi $^{1}$ and Réfrégier et al. ${ }^{2}$ that the interaction between two incident waves can be significantly enhanced by application of dc voltage and detuning of one of the waves. The enhancement was maximum when the moving interference wave was in resonance with the space-charge wave, which is an eigenwave of the system. ${ }^{3}$ A nonresonant excitation employing an applied ac voltage was shown by Stepanov and Petrov $^{4}$ to lead to similar enhancement. More recently, a new kind of resonant interaction ${ }^{5-7}$ was proposed that, instead of detuning, relies on applied dc and ac fields. It was shown in Refs. 5-7 that the resonance took place when the frequency of the applied ac field agreed with the eigenfrequency of the space-charge wave. The purpose of this Letter is to investigate experimentally this new resonant mechanism and to introduce corrections to the model of Ref. 7 that will lead to good agreement between the experimental and the theoretical results.

The experimental arrangement for two-wave mixing is shown schematically in Fig. 1. A frequency-doubled diode-pumped Nd:YAG laser $\left(\lambda=532 \mathrm{~nm}\right.$; TEM $_{00}$ power, $500 \mathrm{~mW}$ ) was used as the light source. A variable beam splitter controlled the intensity ratio of the two beams, which were incident upon the (110) face of a bismuth silicate (BSO) crystal with an interbeam angle of $1.4^{\circ}$, thus producing fringes with approximately $20-\mu \mathrm{m}$ spacing. Both incident beams were linearly polarized normal to the (110) face of the crystal. The external fields were applied across the (001) faces. The crystal dimensions were all $10 \mathrm{~mm}$, mirrors M3 and M4 were mounted upon piezoelectric translators, and, for frequency detuning, sawtooth voltages of opposite polarity were applied to these translators with an amplitude sufficient to cause an optical phase change of $2 \pi$ between the beams. The modulation depth of the interference pattern, $m$, was equal to 0.1 (corresponding to an input intensity ratio of $\sim 100$ ), and a photodetector was used for monitoring the intensity of the weak signal beam. The total intensity of the beams in all the experiments was $I_{0}=5 \mathrm{~mW} \mathrm{~cm}^{-2}$.

According to the predictions of the simple model referred to above, the maximum of the new resonance occurs at the same frequency as that of the resonance obtained when the optical beams are detuned. Hence in our first set of experiments we measured the gain as a function of detuning. The results are shown in Fig. 2 for three different values of the applied dc field. The corresponding theoretical curves were obtained with the theoretical approach of Réfrégier et al. ${ }^{2}$ with the following parameters: density of

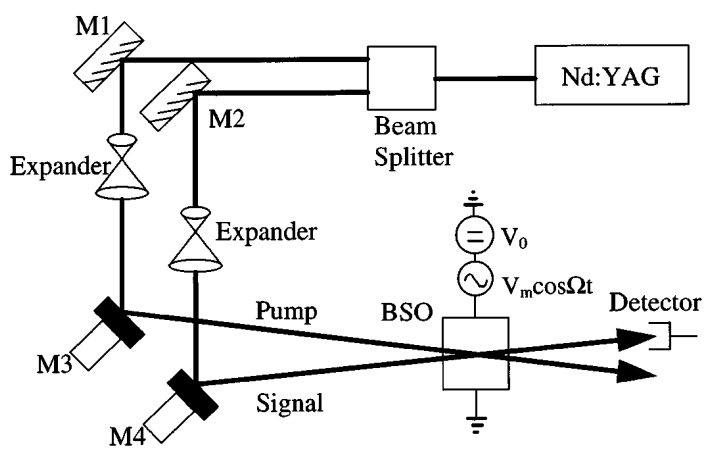

Fig. 1. Experimental arrangement: M1-M4, mirrors. 


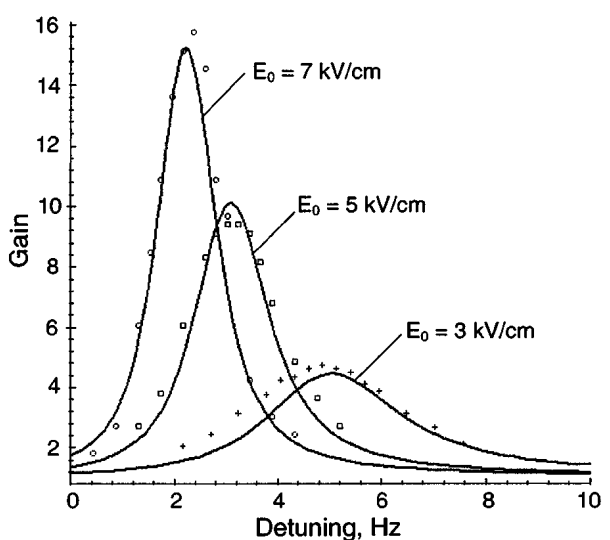

Fig. 2. Two-wave mixing gain as a function of detuning frequency for different values of the dc electric field. Solid curves, theoretical values; points, experimental values.

acceptors $N_{A}=1.6 \times 10^{21} \mathrm{~m}^{-3}$, density of donors $N_{D}=1.22 \times 10^{25} \mathrm{~m}^{-3}$, photoionization constant $s=2.0 \times 10^{-5} \mathrm{~m}^{2} / \mathrm{J}$, electron mobility $\mu=1.2 \times 10^{-6} \mathrm{~m}^{2} / \mathrm{V} \mathrm{s}$, recombination constant $\gamma=1.6 \times 10^{-17} \mathrm{~m}^{3} / \mathrm{s}$, effective electro-optic coefficient $r_{\text {eff }}=1.05 \times 10^{-12} \mathrm{~m} / \mathrm{V}$, and static permittivity $\epsilon_{r}=56$. Note that $r_{\text {eff }}$ is much smaller than that usually given $\left(\sim 4 \times 10^{-12} \mathrm{~m} / \mathrm{V}\right)$ to account for losses and optical activity that were not taken into account in the theory.

In the next set of experiments an ac field of $E_{m}=$ $3 \mathrm{kV} \mathrm{cm}{ }^{-1}$ was used instead of detuning, and three values of the applied dc field were chosen, $E_{0}=$ $3,5,7 \mathrm{kV} \mathrm{cm}^{-1}$. As predicted by the theory ${ }^{7}$ the gain was found to vary in an oscillatory manner as a function of time with the same period as that of the applied ac field. In the actual experiment the ac frequency was slowly changed, leading to a variation in the amplitude of the measured gain. The envelope of this gain function as a function of applied frequency is shown in Fig. 3(a), in which the curves simply connect the points at which the gain was maximum. It can be seen that for the highest dc field there is a clear resonance occurring at $2.2 \mathrm{~Hz}$ that agrees very well with the frequency, yielding resonance for the detuning case. However, for $E_{0}=5 \mathrm{kV} \mathrm{cm}{ }^{-1}$ there is no longer a single peak, and the curve appears hardly to vary for the lowest field, $E_{0}=3 \mathrm{kV} \mathrm{cm}-1$.

The predictions of the simple model can be seen to be correct for the highest dc field, but the approximation deteriorates as the dc field decreases relative to the ac field amplitude, $E_{m}$. Clearly some modifications are needed. Let us recall at this stage the basic assumption of the simple model. The space-charge field was represented there by three terms:

$$
\begin{aligned}
E(x, t)= & 1 / 2\left[E_{s} \exp (j k x)+E_{1} \exp j(k x-\Omega t)\right. \\
& \left.+E_{-1} \exp j(k x+\Omega t)\right]+ \text { c.c. },
\end{aligned}
$$

where $k$ and $\Omega$ are the wave number and the frequency of the space-charge wave, respectively, and $x$ is a coordinate along the direction of the external electric field. $E_{S}, E_{1}$, and $E_{-1}$ represent the amplitudes of the stationary interference pattern and of the forward- and backward-moving space-charge waves, respectively. In the first-order approximation ${ }^{5,7} E_{1}$ and $E_{-1}$ are proportional to $\left(\Omega-\Omega_{1}\right)^{-1}$ and $(\Omega+$ $\left.\Omega_{1}\right)^{-1}$, respectively, where

$$
\begin{aligned}
\Omega_{1}=\Omega_{s}\left(1+j E_{M} / E_{0}\right)^{-1}, \quad \Omega_{s} & =-\left(k \mu \tau_{r} \tau_{d}\right)^{-1}, \\
E_{M} & =-\left(k \mu \tau_{r}\right)^{-1},
\end{aligned}
$$

$\tau_{d}$ is the dielectric relaxation time, and $\tau_{r}$ is the electron lifetime. When $E_{M} \ll E_{0}$ (valid for the parameters chosen), the backward wave can be shown to be negligible except at very small frequencies. On the other hand there are a vast number of higher-order forward-moving waves owing to the nonlinearity of the crystal. We obtain good agreement with our experimental results if we include just one additional wave at the second temporal harmonic with amplitude $E_{2}$. Hence our assumption for the space-charge wave is

$$
\begin{aligned}
E(x, t)= & 1 / 2\left[E_{s} \exp (j k x)+E_{1} \exp j(k x-\Omega t)\right. \\
& \left.+E_{2} \exp j(k x-2 \Omega t)\right]+ \text { c.c. }
\end{aligned}
$$

The mathematical problem is then to solve the nonlinear partial differential equation derived in Refs. 5 and 8:

$$
\begin{aligned}
\left(E_{0}+E_{m} \cos \Omega t+E\right)(\mu & \left.\frac{\partial^{2} E}{\partial x \partial t}+\frac{1}{\tau_{r} \tau_{d}} \frac{I_{\sim}}{I_{0}}\right) \\
& +\frac{1}{\tau_{r} \tau_{d}} E+\frac{1}{\tau_{r}} \frac{\partial E}{\partial t}=0,
\end{aligned}
$$

with the aid of the above assumption [Eq. (3)]. In Eq. (4), $I_{\sim}$ is the component of the light intensity in the
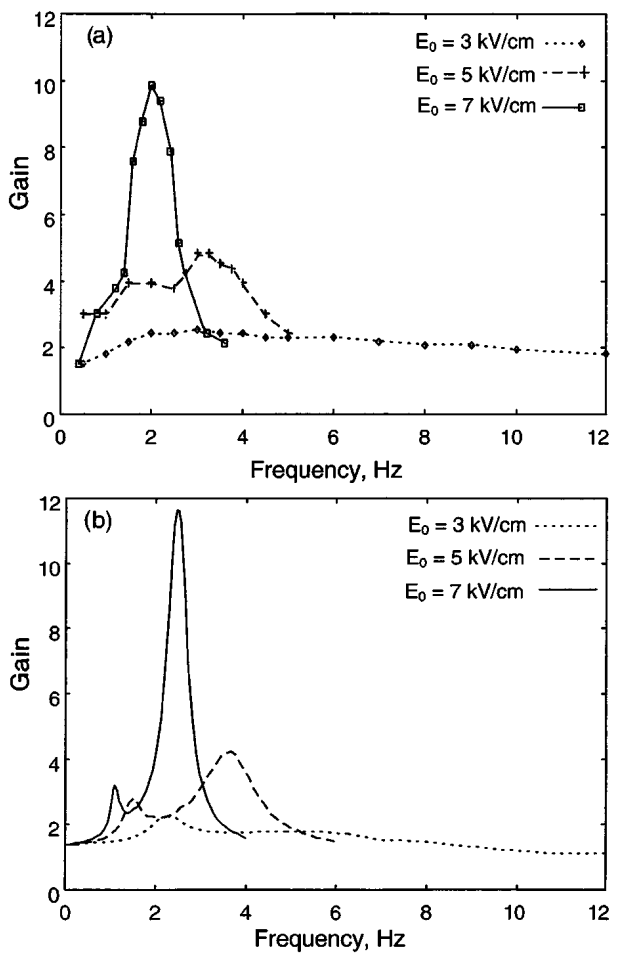

Fig. 3. (a) Experimental and (b) theoretical curves for the maximum two-wave-mixing gain as a function of the ac field frequency for different values of the dc field. $E_{m}=3 \mathrm{kV} / \mathrm{cm}$. 
crystal that varies with the $x$ coordinate. Diffusion of free electrons is neglected, and it is assumed that the total external electric field is much smaller than the maximum achievable space-charge field. The calculations are rather laborious but quite straightforward. Following the same method as in Ref. 7 we obtain the following expressions for the three unknown amplitudes:

$$
\begin{aligned}
E_{1} & =\frac{m E_{m}}{2} \Omega \\
\times & {\left[\Omega-\Omega_{1}-\frac{1}{2} \frac{\Omega^{2}}{\left(1+j E_{M} / E_{0}\right)^{2}\left(2 \Omega-\Omega_{1}\right)}\left|\frac{E_{m}}{E_{0}}\right|^{2}\right]^{-1}, }
\end{aligned}
$$

$E_{2}=-\frac{E_{1}}{2} \frac{E_{m}}{E_{0}} \frac{\Omega}{\left(1+j E_{M} / E_{0}\right)\left(2 \Omega-\Omega_{1}\right)}$,

$E_{s}=-E_{0} m+\frac{1}{2} \frac{E_{m}^{*}}{E_{0}} \frac{\Omega}{\Omega_{s}} E_{1}$.

It can be seen from Eqs. (5)-(7) that $E_{1}$ is resonant at $\Omega=\Omega_{s}$, the eigenfrequency of the space-charge wave, provided that $E_{M} \ll E_{0}$ and $E_{m}$ is small in comparison with $E_{0}$. Since both $E_{2}$ and $E_{s}$ are proportional to $E_{1}$, those amplitudes will also depend on $\Omega$ in a resonant manner. However, there is an additional resonance of $E_{2}$ at $\Omega=\Omega_{s} / 2$, as can be seen from Eq. (6). Owing to the nonlinear coupling between $E_{1}$ and $E_{2}$, this additional resonance causes the appearance of a lowfrequency peak in $E_{1}$ and also an increase of the position of the high-frequency peak as $E_{m} / E_{0}$ increases.

To compare this theory with the experimental results, we need to calculate the envelope of the gain function:

$$
\begin{aligned}
G= & \max \left(\operatorname { e x p } \left\{\frac { r _ { \text { eff } } \epsilon _ { r } ^ { 3 / 2 } k L } { 2 m } \operatorname { I m } \left[E_{s}+E_{1} \exp (-j \Omega t)\right.\right.\right. \\
& \left.\left.\left.+E_{2} \exp (-2 j \Omega t)\right]\right\}\right) .
\end{aligned}
$$

The theoretical curves for the parameters given above can now be plotted [Fig. 3(b)]. There is no doubt that the theoretical curves for the lower values of the applied dc field show now the general features of the experimental results, namely, the additional low-frequency resonance and the shifting of the highfrequency peak. Note that the values well below the lower peak are inaccurate owing to neglect of the backward-moving wave.

More-accurate theoretical results could be obtained by addition of extra terms, that is, higher temporal harmonics, to our assumed solution of Eq. (4), although the amount of algebra would soon become prohibitive for an analytical solution. What we have discussed so far applies to the case in which the small modulation assumption $(m \ll 1)$ is valid. As the modulation increases, the higher spatial harmonics would also have to be taken into account, i.e., the space-charge field should be, strictly speaking, assumed to be in the form

$E(x, t)=1 / 2 \sum_{p, q=-\infty}^{\infty} E_{p, q} \exp j(p k x-q \Omega t)+$ c.c.

Analytical solutions are no longer possible in this case, but numerical methods might still work for a truncated set. It may be worth mentioning here that, in the high-modulation solution of Au and Solymar, ${ }^{9}$ for the detuning resonance the numerical problems were successfully solved for numbers of spatial harmonics greater than 20 .

The new resonance proposed in Ref. 5-7 has been shown to behave according to theoretical predictions for large applied dc fields. Experimental results obtained for lower dc fields have also been matched with a modified theory.

\section{References}

1. J. P. Huignard and A. Marrakchi, Opt. Commun. 38, 249 (1981).

2. Ph. Réfrégier, L. Solymar, H. Rajbenbach, and J. P. Huignard, J. Appl. Phys. 57, 45 (1985).

3. R. F. Kazarinov, R. A. Suris, and B. I. Fuks, Sov. Phys. Semicond. 6, 500 (1972).

4. S. I. Stepanov and M. P. Petrov, Opt. Commun. 53, 292 (1985).

5. V. A. Kalinin, Proc. SPIE 2051, 582 (1993).

6. V. S. Liberman and B. Ya. Zel'dovich, Int. J. Non-linear Opt. Phys. 3, 39 (1994).

7. V. A. Kalinin and L. Solymar, Appl. Phys. Lett. 68, 197 (1996).

8. L. Solymar, D. J. Webb, and A. Grunnet-Jepsen, Prog. Quantum Electron. 18, 377 (1994).

9. L. B. Au and L. Solymar, J. Opt. Soc. Am. A 7, 1554 (1990). 